\title{
Motifs du recours aux services d'urgence des principaux hôpitaux du Grand Tunis
}

\author{
H.L. Ben Gobrane, ${ }^{7}$ H. Aounallah-Skhiri, ${ }^{1}$ A. Ben Hamida, ${ }^{2}$ N. Somrani, ${ }^{3}$ M. Ayachi, ${ }^{1}$ N. Achour ${ }^{1}$ et M. Hsairi ${ }^{1}$
}

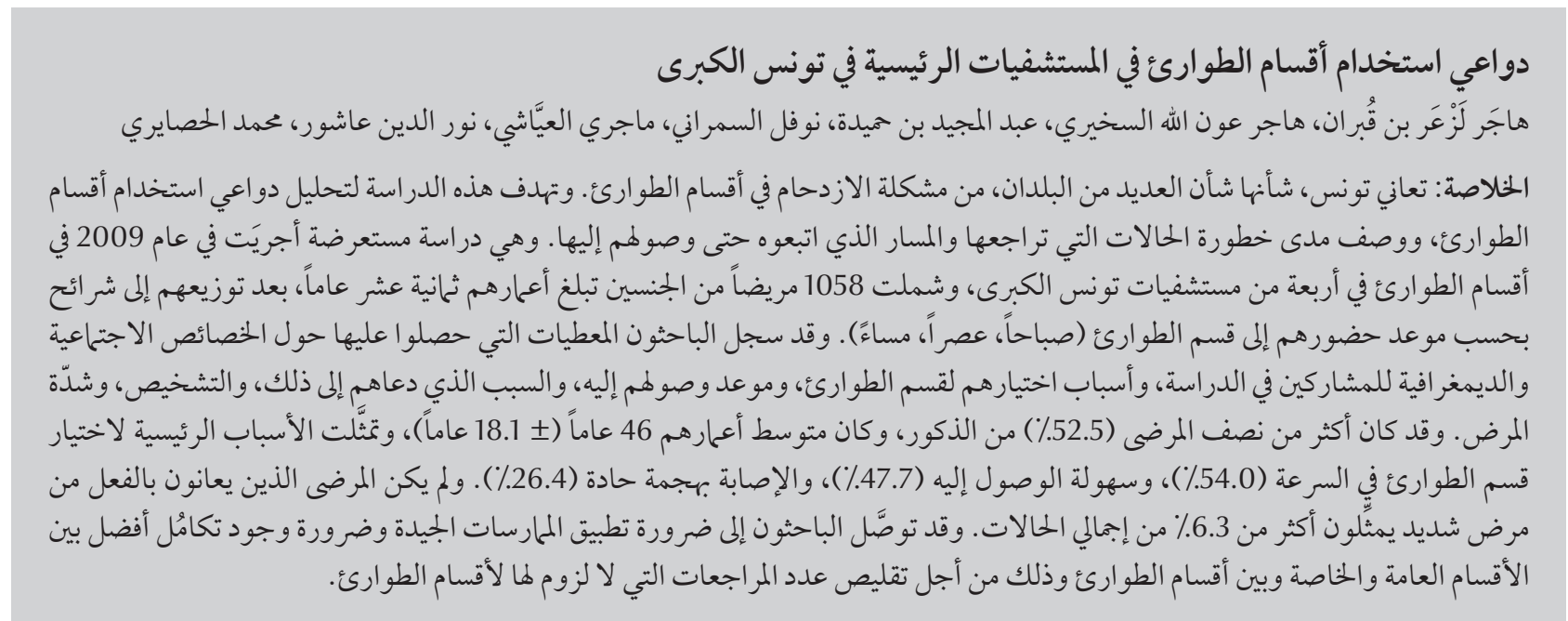

RÉSUMÉ La Tunisie, à l'instar de nombreux pays, n'est pas épargnée par le problème de l'encombrement majeur des services d'urgence. Cette étude analyse les motifs de recours aux urgences et décrit leur degré de gravité et l'itinéraire que les usagers de ces services empruntent avant leur arrivée. Il s'agit d'une étude transversale effectuée en 2009 dans les services d'urgence de quatre hôpitaux du Grand Tunis, auprès d'un échantillon de 1058 patients des deux sexes, âgés de 18 ans et plus, avec une stratification selon l'horaire de recours à ces services (matin, après-midi et soir). Des données ont été recueillies sur les caractéristiques socio-démographiques des participants, les raisons du choix du service, l'heure et les motifs de consultation, le diagnostic et la gravité de l'état de santé. Plus de la moitié des patients (52,5\%) étaient de sexe masculin ; la moyenne d'âge était de 46,0 ans (ET 18,1). Les raisons du choix du service d'urgence étaient surtout la rapidité $(54,0 \%)$ et la facilité d'accès $(47,7 \%)$, suivies de la survenue d'un épisode aigu (26,4\%). Les patients se trouvant dans un état réellement grave ne représentaient que 6,3\% des cas. De bonnes pratiques et une meilleure complémentarité entre les services publics et privés et les services d'urgence sont nécessaires pour réduire le recours inutile à ces derniers.

\section{Reasons for using emergency departments of major hospitals in Greater Tunis}

ABSTRACT Tunisia, similar to many countries, has a problem of overcrowding of the emergency departments (ED). This study aimed to analyse the reasons for using EDs, and to describe the seriousness of the attendees' condition and their itinerary before their arrival at ED. This cross-sectional study in 2009 was conducted in ED of 4 hospitals in Greater Tunis and targeted 1058 patients of both sexes, aged $\geq 18$ years, with stratification according to time of day of presentation to ED (morning, afternoon and evening). Information was recorded on the sociodemographic characteristics of the study participants, reasons for choosing ED, time of and reason for consultation, diagnosis and severity of illness. Over half the patients (52.5\%) were male and the mean age was 46.0 (SD 18.1) years. The main reasons for choosing the ED were: speed (54.0\%) and ease of access (47.7\%) of ER and occurrence of an acute episode (26.4\%). Patients with serious illness accounted for only $6.3 \%$ of those interviewed. Implementation of good practices and better coordination between public and private services and the ED are needed to reduce unnecessary visits to ED.

${ }^{1}$ Institut National de Santé Publique, Tunis (Tunisie) (Correspondance à adresser à H.L. Ben Gobrane : hadi_cherifa@yahoo.fr).

2 Faculté de Médecine de Tunis, Tunis (Tunisie).

${ }^{3}$ Ministère de la Santé publique, Tunis (Tunisie).

Reçu : 18/04/11; accepté : 20/04/11 


\section{Introduction}

Les services d'urgence (SU) du monde entier constituent un maillon vital entre la prise en charge préhospitalière et l'hospitalisation des consultants [1-4].

Au cours des dernières décennies, les SU des hôpitaux n'ont cessé de voir leur activité augmenter [2]. Cette hausse constante de la fréquentation des services d'urgence est un phénomène commun à tous les pays qui en possèdent [3,4]. Au Canada en 2001, on enregistrait une augmentation du nombre de passages annuels aux urgences de $5 \%$ en 3 ans [5]; au Royaume-Uni, on a observé une augmentation de $1 \%$ par an depuis le milieu des années 1990 [6], alors qu' aux États-Unis, une croissance de $14 \%$ a été enregistrée entre 1992 et 1999 [7]. Par ailleurs, plusieurs pays de la Région de la Méditerranée orientale sont concernés par cette hausse du recours aux $S U[8,9]$. À titre d'exemple, entre les années 2003 et 2005, l'Arabie saoudite a connu une augmentation du nombre de passages aux urgences de $30 \%$ [1].

La Tunisie, à l'instar de nombreux pays, n'est pas épargnée par le problème de l'encombrement majeur des services d'urgence : au total, plus de 3500000 recours sont enregistrés chaque année [3]. Une structure universitaire reçoit en moyenne plus de 100000 cas par an. Ces chiffres indiquent une confrontation de ces services à un niveau de charge structurellement tendu, qui est préjudiciable au bon fonctionnement des urgences.

Parmi les causes responsables de cet encombrement figure l'absence de caractère urgent du motif de recours [2]. En Tunisie, peu d'études se sont attardées sur les motifs de recours aux services d'urgence et sur les trajectoires que les usagers ont empruntées avant leur arrivée à ces services. C'est l'objet de cette étude qui se propose d'analyser les motifs de recours aux urgences et la gravité de l'état de santé des patients.
Elle décrit également l'itinéraire que les usagers des SU empruntent avant leur arrivée aux urgences.

\section{Méthodologie}

Il s'agit d'une étude transversale qui a été effectuée dans les services d'urgence des hôpitaux La Rabta, Charles Nicolle, Habib Thameur et Ben Arous.

\section{Échantillonnage}

Ce travail a ciblél'ensemble des patients des deux sexes, âgés de 18 ans et plus, quel que soit l'horaire de recours aux services d'urgence des hôpitaux susmentionnés durant les mois de septembre et octobre 2009.

La taille de l'échantillon a été estimée à 1000 personnes, réparties sur les quatre hôpitaux, choisies selon un sondage systématique à partir du registre d'inscription en incluant d'une manière prospective les malades dont le numéro est impair, et jusqu'à l'atteinte de l'effectif prévu, avec une stratification sur les plages horaires (séance du matin de $7 \mathrm{~h}$ à $12 \mathrm{~h} 59$, de l'après-midi de $13 \mathrm{~h}$ à 18 h 59, du soir de 19 hà 6 h 59).

\section{Recueil des données}

Le recueil des données a été effectué par questionnaire, préalablement testé et administré par des enquêteurs infirmiers.

Les données concernaient les caractéristiques socio-démographiques, les raisons ayant motivé le choix du service d'urgence, l'heure et les motifs de consultation, l'établissement d'un diagnostic avec le cas échéant, la nature du diagnostic et la gravité de l'état de santé, en se fondant sur la classification clinique des malades des urgences (CCMU) [6].

\section{Saisie et analyse des données}

Les données ont été saisies à l'aide du logiciel Epi info (version 6.04). L'analyse a été effectuée avec le logiciel SPSS version 10.
Les variables quantitatives ont été décrites par les paramètres classiques (moyenne, écart type [ET]), et les variables qualitatives, par un calcul de fréquences. Tous les tests statistiques sont bilatéraux et le seuil de signification a été fixé à 0,05 . La comparaison des moyennes a été effectuée en utilisant le test $t$ de Student et le test d'analyse de variance. La comparaison des pourcentages a été effectuée par le test de $\chi^{2}$ et le test exact de Fisher.

\section{Résultats}

\section{Caractéristiques socio- démographiques}

Au total, 1058 patients ont participé à cette étude. Ils étaient répartis de façon sensiblement équivalente entre les quatre hôpitaux sélectionnés. Plus de la moitié des patients ( $52,5 \%$ ) étaient de sexe masculin; la moyenne d'âge était de 46,0 ans (ET 18,1); 24,8\% étaient âgés de plus de 60 ans. La majorité des patients $(61,0 \%)$ étaient mariés et $28,0 \%$ étaient célibataires. Il s'agit d'une population relativement peu instruite, dont près de la moitié (45,4\%) n’a pas dépassé le cycle de l'enseignement primaire. Environ la moitié des participants $(46,6 \%)$ exerçait une activité professionnelle ; 32,1\% étaient sans activité ou femme au foyer et 12,5\% étaient retraités. La répartition selon le régime d'assurance maladie montre que $52,0 \%$ bénéficiaient du régime de la Caisse nationale d'Assurance maladie $(\mathrm{CNAM})$ « Soins dans les structures publiques $\gg ; 17,4 \%$ étaient des indigents et 18,1\% étaient des malades payants.

\section{Répartition selon I'hôpital et la plage horaire}

Le recours aux urgences était plus fréquent au cours de la nuit (40,2\%), notamment pour les hôpitaux Habib Thameur $(48,0 \%)$ et Ben Arous $(43,6 \%)$ (cf. tableau 1). 


\begin{tabular}{|c|c|c|c|c|c|c|}
\hline \multirow[t]{3}{*}{ Heure } & \multicolumn{6}{|c|}{ Hôpital } \\
\hline & & La Rabta & Charles Nicolle & Habib Thameur & Ben Arous & Total \\
\hline & $n$ & $\%$ & $\%$ & $\%$ & $\%$ & $\%$ \\
\hline Nuit & 425 & 32,5 & 36,3 & 48,0 & 43,6 & 40,2 \\
\hline Matin & 365 & 45,4 & 38,8 & 24,2 & 30,0 & 34,5 \\
\hline Après-midi & 268 & 22,1 & 24,9 & 27,9 & 26,4 & 25,3 \\
\hline Total & 1058 & 100,0 & 100,0 & 100,1 & 100,0 & 100,0 \\
\hline
\end{tabular}

\section{Accessibilité des services d'urgence et moyen de transport}

Plus de la moitié des participants $(55,4 \%)$ résidait à moins de $5 \mathrm{~km} \mathrm{du}$ service d'urgence et $69,1 \%$ à moins de 30 minutes. L'accessibilité aux services d'urgence était différente selon l'hôpital, le service de l'hôpital Habib Thameur ayant été déclaré le plus accessible par les patients interrogés.

Le principal moyen de transport utiliséétait deloin « unevoiture privée » $(79,8 \%)$; le transport par ambulance ne représentait que $2,4 \%$ des cas et le transport par la protection civile n'a été assuré que dans $2,8 \%$ des cas.

\section{Modalités d'orientation vers le service d'urgence}

Le recours au service d'urgence se faisait selon une initiative personnelle dans environ trois quarts des cas $(72,9 \%)$; le patient n'était adressé par un médecin que dans $17,4 \%$ des cas et dans $6,4 \%$ des cas, il était adressé par un autre service d'urgence. La fréquence de recours préalable à un médecin augmente avec l'âge de façon significative, passant de $11,8 \%$ chez les patients de moins de 25 ans à $26,4 \%$ chez les patients de plus de 70 ans. Elle est relativement faible chez les personnes célibataires $(12,5 \%)$ mais beaucoup plus élevée chez les personnes divorcées (42,9\%). Le recours préalable à un médecin est aussi significativement plus fréquent durant la séance du matin (23,8\%), par rapport aux séances de l'après-midi et de la nuit ( $14,2 \%$ et $13,9 \%$ respectivement, $\mathrm{p}<0,005)$ (cf. tableau 2).

\section{Principales raisons ayant motivé le choix du service d'urgence}

Les raisons majeures du choix du service d'urgence étaient la rapidité (54,0\%) et la facilité d'accès au service d'urgence $(47,7 \%)$, notamment chez les personnes en activité, suivies de la survenue d'un épisode aigu (26,4\%). La disponibilité des équipements a été indiquée dans $12,4 \%$ des cas et le manque d'argent dans $10,3 \%$, alors que l'absence du médecin traitant n'a été mentionnée que par 3,3\% des patients. Ces motifs ont été plus fréquemment évoqués par les personnes d'un niveau de scolarisation inférieur au cycle primaire (cf. tableau 3).

\section{Symptomatologie ayant motivé le recours aux urgences et état de gravité des patients}

Pour la majorité des patients interrogés $(81,0 \%)$, il s'agissait d'un problème de santé intercurrent, principalement un problème digestif $(16,7 \%)$, un problème respiratoire $(15,7 \%)$ ou un problème cardiovasculaire $(15,4 \%)$, alors que pour les autres patients (19,3\%), il s'agissait d'une douleur aiguë ou d'une blessure, en particulier un accident domestique (7,0 \%), un acte de violence $(4,4 \%)$ ou un accident de la voie publique (3,9\%).

Les accidents domestiques étaient relativement plus fréquents chez les patients de sexe féminin consultant à l'hôpital Charles Nicolle. Les accidents deviolencequantà euxétaientbeaucoup plus fréquents chez les patients de sexe masculin, relativement jeunes (âgés de moins de 40 ans), consultant la nuit à l'hôpital Ben Arous.

Les problèmes digestifs étaient plus fréquents chez les femmes en consultation àl'hôpital Habib Thameur. Au contraire, les problèmes respiratoires et cardiovasculaires concernaient davantage les hommes. Ces problèmes cardiovasculaires étaient relativement plus fréquents chez les patients de plus de 60 ans (cf. tableau 4).

Les patients se trouvant dans un état réellement grave ne représentaient que $6,3 \%$ des cas. Aucune gravité de l'état de santé n'a été observée chezle tiers des patients environ $(32,8 \%)$; pour plus de la moitié, l'état de santé était stable mais des examens complémentaires étaient recommandés (cf. tableau 5).

\section{Discussion}

Le phénomène d'encombrement des urgences semble universel. Il s'explique par des causes communes à tous les pays, et par des causes spécifiques dues à la politique sanitaire $[5,6]$. La décision du choix du recours aux SU varie notamment selon le niveau socioéconomique, l'âge et l'horaire d'arrivée aux urgences $[7,10]$.

La clientèle des services d'urgence dans notre étude était essentiellement composée de patients de niveau d'instruction moyen ; près de la moitié n'avait pas d'emploi. Ce profil de consultants se rapproche de celui d'une étude canadienne qui a observé que les patients à plus faible revenu ont été plus nombreux à se rendre dans les $\mathrm{SU}$ 


\begin{tabular}{|c|c|c|c|c|}
\hline \multirow[t]{2}{*}{ Variable } & \multirow[t]{2}{*}{ Nombre } & \multirow{2}{*}{$\begin{array}{c}\text { Initiative personnelle } \\
\qquad \%\end{array}$} & \multirow{2}{*}{$\begin{array}{l}\text { Adressé par un } \\
\text { médecin } \\
\%\end{array}$} & \multirow{2}{*}{$\begin{array}{c}\text { Adressé par un autre } \\
\text { service d'urgence } \\
\%\end{array}$} \\
\hline & & & & \\
\hline \multicolumn{5}{|l|}{$\hat{A g e}$ (ans) } \\
\hline$<25$ & 144 & 75,0 & 11,8 & 6,9 \\
\hline $25-39$ & 274 & 75,5 & 13,5 & 6,6 \\
\hline $40-49$ & 208 & 77,9 & 14,4 & 4,8 \\
\hline $50-59$ & 169 & 71,0 & 19,5 & 6,5 \\
\hline $60-69$ & 119 & 68,1 & 24,4 & 5,0 \\
\hline 70 et + & 144 & 64,6 & 26,4 & 9,0 \\
\hline$p$ & & 0,05 & $<0,01$ & 0,7 \\
\hline \multicolumn{5}{|l|}{ Sexe } \\
\hline Masculin & 555 & 71,5 & 16,0 & 7,7 \\
\hline Féminin & 503 & 74,4 & 18,9 & 5,0 \\
\hline$p$ & & 0,3 & 0,22 & 0,06 \\
\hline \multicolumn{5}{|l|}{ État civil } \\
\hline Célibataire & 296 & 75,0 & 12,5 & 7,1 \\
\hline Marié(e) & 645 & 73,3 & 18,3 & 5,6 \\
\hline Divorcé(e) & 7 & 57,1 & 42,9 & 0,0 \\
\hline Veuf(ve) & 110 & 65,5 & 23,6 & 10,0 \\
\hline$p$ & & 0,19 & $<0,01$ & 0,28 \\
\hline \multicolumn{5}{|l|}{ Niveau de scolarisation } \\
\hline Analphabète & 324 & 72,2 & 20,1 & 6,2 \\
\hline Primaire & 157 & 73,9 & 15,3 & 8,9 \\
\hline Collège & 128 & 64,8 & 22,7 & 7,0 \\
\hline Secondaire & 312 & 75,0 & 14,4 & 5,4 \\
\hline Universitaire & 137 & 75,9 & 15,3 & 5,8 \\
\hline$p$ & & 0,22 & 0,14 & 0,67 \\
\hline \multicolumn{5}{|l|}{ Profession } \\
\hline En activité & 493 & 74,6 & 15,8 & 5,9 \\
\hline Retraité(e) & 132 & 65,2 & 22,7 & 9,1 \\
\hline Étudiant(e) & 94 & 78,7 & 12,8 & 5,3 \\
\hline Sans activité & 82 & 65,9 & 14,6 & 11,0 \\
\hline Femme au foyer & 257 & 73,5 & 20,2 & 5,1 \\
\hline$p$ & & 0,07 & 0,15 & 0,22 \\
\hline \multicolumn{5}{|l|}{ Couverture sociale } \\
\hline Indigent & 183 & 74,3 & 20,8 & 4,4 \\
\hline Soins dans les structures publiques ${ }^{\mathrm{a}}$ & 550 & 74,5 & 17,5 & 6,9 \\
\hline Médecin de famille ${ }^{a}$ & 6 & 83,3 & 16,7 & 0,0 \\
\hline Remboursement des frais ${ }^{\mathrm{a}}$ & 30 & 73,3 & 23,3 & 3,3 \\
\hline Payant & 190 & 66,3 & 14,2 & 7,9 \\
\hline Autre & 98 & 73,5 & 14,3 & 6,1 \\
\hline$p$ & & 0,34 & 0,46 & 0,69 \\
\hline \multicolumn{5}{|l|}{ Heure de consultation } \\
\hline Nuit & 425 & 74,8 & 13,9 & 7,1 \\
\hline Matin & 365 & 66,3 & 23,8 & 6,8 \\
\hline Après-midi & 268 & 78,7 & 14,2 & 4,9 \\
\hline$p$ & & $<0,01$ & $<0,001$ & 0,47 \\
\hline Total & 1058 & 72,9 & 17,4 & 6,4 \\
\hline
\end{tabular}

${ }^{a}$ Régime CNAM (Caisse nationale d'Assurance maladie) 


\begin{tabular}{|c|c|c|c|c|c|c|c|c|c|}
\hline \multirow[t]{2}{*}{ Variable } & \multirow[t]{2}{*}{ n } & \multicolumn{8}{|c|}{ Raisons du choix du recours aux urgences (\%) } \\
\hline & & 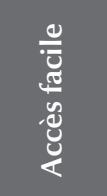 & $\begin{array}{l}\frac{0}{\circ} \\
\frac{0}{2} \\
\frac{2}{8} \\
\frac{8}{8} \\
\frac{8}{4}\end{array}$ & 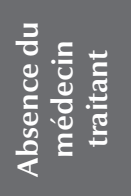 & 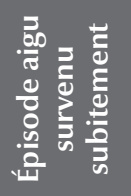 & 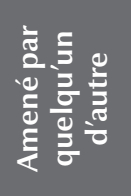 & 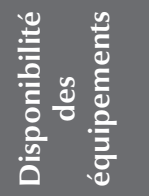 & 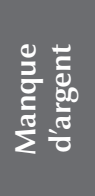 & 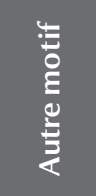 \\
\hline \multicolumn{10}{|l|}{ Âge (ans) } \\
\hline$<25$ & 144 & 47,9 & 51,4 & 7,6 & 19,4 & 17,5 & 8,3 & 10,4 & 3,5 \\
\hline $25-39$ & 274 & 46,4 & 53,3 & 4,7 & 23,0 & 16,8 & 12,8 & 9,5 & 6,6 \\
\hline $40-49$ & 208 & 58,7 & 63,5 & 1,4 & 26,0 & 11,5 & 10,1 & 11,1 & 1,9 \\
\hline $50-59$ & 169 & 44,4 & 52,7 & 1,8 & 27,8 & 11,8 & 13,6 & 10,7 & 7,7 \\
\hline $60-69$ & 119 & 42,0 & 47,1 & 3,4 & 37,0 & 9,2 & 16,0 & 9,2 & 5,0 \\
\hline 70 et + & 144 & 43,1 & 51,7 & 0,7 & 29,9 & 10,4 & 14,6 & 11,1 & 6,3 \\
\hline$p$ & & 0,01 & 0,05 & $<0,01$ & 0,02 & 0,12 & 0,35 & 0,98 & 0,11 \\
\hline \multicolumn{10}{|l|}{ Sexe } \\
\hline Masculin & 555 & 49,2 & 52,7 & 2,9 & 25,2 & 16,4 & 11,5 & 9,9 & 5,6 \\
\hline Féminin & 503 & 46,1 & 55,5 & 3,8 & 27,6 & 10,0 & 13,3 & 10,7 & 4,8 \\
\hline$p$ & & 0,31 & 0,36 & 0,41 & 0,37 & $<0,01$ & 0,37 & 0,66 & 0,55 \\
\hline \multicolumn{10}{|l|}{ État civil } \\
\hline Célibataire & 296 & 45,6 & 50,3 & 5,4 & 19,6 & 18,0 & 9,1 & 9,5 & 5,1 \\
\hline Marié(e) & 645 & 50,9 & 57,1 & 2,9 & 27,9 & 11,8 & 11,9 & 10,7 & 4,7 \\
\hline Divorcé(e) & 7 & 42,9 & 42,9 & 0,0 & 42,9 & 28,6 & 28,6 & 0,0 & 14,3 \\
\hline Veuf(ve) & 110 & 35,5 & 46,4 & 0,0 & 34,5 & 9,1 & 22,7 & 10,9 & 8,2 \\
\hline$p$ & & 0,02 & 0,07 & 0,04 & $<0,01$ & 0,02 & 0,001 & 0,75 & 0,31 \\
\hline \multicolumn{10}{|l|}{ Niveau de scolarisation } \\
\hline Analphabète & 324 & 52,5 & 55,1 & 1,9 & 27,2 & 12,0 & 15,4 & 18,5 & 5,2 \\
\hline Primaire & 157 & 54,1 & 60,5 & 3,2 & 27,4 & 8,9 & 15,3 & 9,6 & 6,4 \\
\hline Collège & 128 & 42,2 & 49,2 & 3,1 & 25,8 & 18,1 & 10,2 & 5,5 & 7,0 \\
\hline Secondaire & 312 & 45,8 & 54,5 & 3,8 & 25,3 & 15,4 & 10,9 & 6,1 & 2,6 \\
\hline Universitaire & 137 & 38,7 & 47,4 & 5,8 & 26,3 & 12,4 & 7,3 & 5,8 & 8,0 \\
\hline$p$ & & 0,01 & $<0,01$ & 0,06 & 0,04 & $<0,01$ & $<0,001$ & 0,01 & 0,09 \\
\hline \multicolumn{10}{|l|}{ Profession } \\
\hline En activité & 493 & 51,1 & 59,6 & 2,8 & 25,2 & 13,2 & 9,7 & 8,9 & 4,7 \\
\hline Retraité(e) & 132 & 42,4 & 48,5 & 3,0 & 32,6 & 12,9 & 11,4 & 6,1 & 6,1 \\
\hline Étudiant(e) & 94 & 47,9 & 43,6 & 6,4 & 16,0 & 22,3 & 6,4 & 7,4 & 4,3 \\
\hline Sans activité & 82 & 31,7 & 37,0 & 7,3 & 31,7 & 22,0 & 13,4 & 18,3 & 6,1 \\
\hline Femme au foyer & 257 & 49,0 & 55,3 & 1,9 & 27,6 & 7,8 & 19,8 & 13,6 & 5,8 \\
\hline$p$ & & 0,01 & $<0,01$ & 0,06 & 0,04 & $<0,01$ & $<0,001$ & 0,01 & 0,9 \\
\hline \multicolumn{10}{|l|}{ Couverture sociale } \\
\hline Indigent & 184 & 48,4 & 54,9 & 2,7 & 25,0 & 10,3 & 17,9 & 13,6 & 11,4 \\
\hline $\begin{array}{l}\text { Soins dans les structures } \\
\text { publiques }\end{array}$ & 550 & 50,2 & 55,2 & 3,6 & 28,4 & 10,0 & 12,7 & 10,0 & 3,1 \\
\hline Médecin de famille & 6 & 50,0 & 50,0 & 16,7 & 16,7 & 0,0 & 16,7 & 0,0 & 0,0 \\
\hline Remboursement des frais & 30 & 60,0 & 46,7 & 6,7 & 26,7 & 6,7 & 6,7 & 10,0 & 6,7 \\
\hline Payant & 192 & 40,1 & 49,0 & 3,6 & 23,4 & 24,1 & 8,3 & 8,3 & 4,7 \\
\hline Autre & 96 & 43,8 & 58,3 & 0,0 & 24,0 & 19,8 & 9,4 & 10,4 & 6,3 \\
\hline$p$ & & 0,14 & 0,58 & 0,14 & 0,74 & $<0,001$ & 0,07 & 0,59 & $<0,01$ \\
\hline Total & 1058 & 47,7 & 54,0 & 3,3 & 26,4 & 13,3 & 12,4 & 10,3 & 5,2 \\
\hline
\end{tabular}




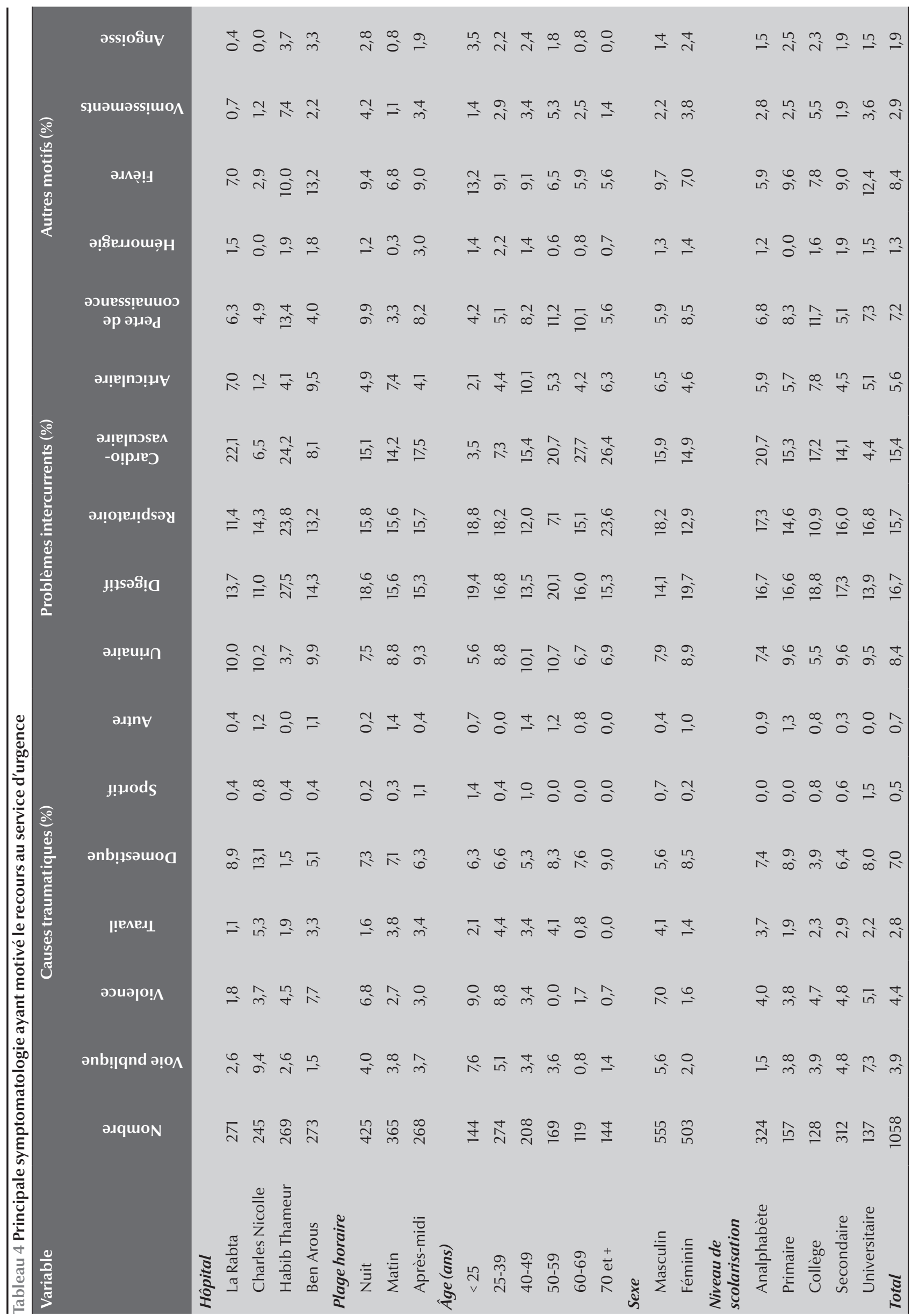




\begin{tabular}{lc}
\hline Tableau 5 Répartition des patients selon leur état de gravité & \\
\hline État de gravité & $\%(\boldsymbol{n}=\mathbf{1 0 5 8})$ \\
PV stable + indication d'actes ou d'examens & 60,8 \\
PV stable & 32,8 \\
PV susceptible de s'aggraver & 5,1 \\
PV engagé & 1,0 \\
PV engagé indication de réanimation aux urgences & 0,3 \\
\hline
\end{tabular}

$P V=$ pronostic vital.

que les patients au revenu plus élevé (18\% contre $13 \%$ ) et que les patients des zones rurales ont davantage recours aux $\mathrm{SU}$ que ceux des zones urbaines (15\% contre $13 \%$ ) [5]. Au contraire en France, les personnes qui ont eu le plus recours aux urgences étaient plutôt bien insérées socialement, et avaient une activité professionnelle $[11,12]$; de même au Koweït, les patients les plus instruits et ceux issus d'une classe sociale élevée étaient les plus nombreux à recourir aux urgences pour des motifs non urgents [13].

Le choix d'un recours aux urgences plus fréquent pour les populations les plus défavorisées serait selon les patients, justifié par le fait que ces derniers pourraient bénéficier d'examens complémentaires, voire d'une consultation spécialisée à moindre coût comparativement au circuit de consultation dans le système ambulatoire.

Dans notre étude, la majorité des consultants ont eu recours aux urgences de leur propre initiative. Ce constat est rapporté par plusieurs études [14-16]. Par ailleurs, le recours aux urgences dans notre étude était prédominant en début de soirée, entraînant un engorgement de ces services. En effet, les patients préfèreraient pour des problèmes de santé non véritablement urgents, recourir aux structures de soins après avoir quitté leur lieu de travail [17]. Ce choix de consulter les SU en dehors des heures d'activité des cabinets médicaux a été rapporté par plusieurs auteurs $[15,18]$. Cette situation serait liée très probablement à l'inadéquation pour la population des horaires de fonctionnement des structures de première ligne.

Notre étude a mis en évidence que le recours aux urgences était largement justifié par des problèmes de santé intercurrents sans gravité, alors qu'une douleur aiguë ou une blessure était assez peu rapportée. L'engorgement des SU dû à des consultations pour une raison de santé non urgente a été aussi relevé dans plusieurs études [14-16] et constitue une réelle préoccupation. Au Koweit, Shah et al. [13] rapportent que $61 \%$ des patients consultaient les SU pour un motif non urgent, alors que ce taux atteignait $70 \%$ en Arabie saoudite [14] et près de $90 \%$ en Jordanie [19].

D'après l'étude effectuée par Gentile [17], une large proportion des personnes interrogées $(35,4 \%)$ a affirmé quil était plus facile et commode de venir directement aux urgences, tandis que $23,1 \%$ ont déclaré que leur médecin traitant était indisponible. Le patient, par sa connaissance de l'offre de soins, choisit le SU pour la présence du plateau technique et sait qu'en dépit du temps d'attente, les SU demeurent en termes d'efficacité le meilleur compromis pour les usagers des urgences $[7,12,15]$.

Enfin, pour un certain nombre de patients (19\%), la recherche de solutions avant le recours au SU s'est avérée inefficace [16]. Les patients préfereraient aussi recourir à l'hôpital plutôt qu'à une autre structure de soins $[13,15]$. En effet, la médecine ambulatoire n'est pas perçue par les consultants des urgences comme étant capable de répondre à une prise en charge rapide et efficace selon plusieurs études [20-22] en raison, d'une part, de l'indisponibilité des médecins de ville et d'autre part, des délais importants pour l'obtention d'un rendez-vous de consultation. Une étude américaine confirme que le recours inapproprié aux urgences était plus fréquent chez les patients considérant que leur système de soins ambulatoires ne peut répondre à leurs attentes [23]. Nos résultats concordent aussi avec ceux observés chez les patients recourant en urgence à la médecine de première ligne dans la région française des Pays de la Loire, dans lesquels les affections aiguës représentent $87 \%$ des motifs de consultation[18], alors que les problèmes traumatiques n'expliquent que $12 \%$ des recours. Tandis que dans les SU hospitaliers, les motifs de recours répertoriés par les médecins se répartissent à part égale entre les problèmes traumatiques et somatiques.

Cette situation est répandue aussi bien dans les pays développés que dans les pays en développement; elle serait due à la défaillance de la médecine de première ligne dans les secteurs public et privé, et la médecine d'urgence comblerait les lacunes de la médecine de première ligne $[21,22]$. En effet, une enquête réalisée dans la région Provence-Alpes-Côte d'Azur en France confirme que dans la majorité des cas, les motifs de consultation auraient pu être gérés dans le circuit ambulatoire [18]. Par ailleurs, selon une étude réaliséee dans 30 centres de soins de santé primaires du district d'Abha dans la région d'Asir (Arabie saoudite), 40,4 \% des médecins ne considéraient pas la majorité des cas comme de véritables urgences [24].

Par ailleurs, la transition épidémiologique et démographique que connaissent la Tunisie ainsi que plusieurs pays en développement $[19,25]$ depuis ces dernières décennies risque d'aggraver le problème de l'engorgement des $\mathrm{SU}$ en raison d'une 
augmentation du recours aux urgences par les patients âgés, souvent porteurs de maladies chroniques, nécessitant des hospitalisations. Ceci est d'autant plus probable que dans la majorité des pays développés, la proportion des personnes âgées fréquentant les SU augmente d'une manière plus importante que leur évolution démographique [26].

Plusieurs études ont rapporté des actions menées pour tenter de remédier à cet engorgement ; certaines ont été jugées efficaces, d'autres moins [20]. Parmi les initiatives qui ont montré une réelle efficacité en termes de réduction du délai d'attente, le développement des soins primaires semble être une solution efficace pour réduire la fréquentation des SU pour motif non urgent [27-30]. En effet, plusieurs études ont rapporté la diminution de la fréquentation des SU par les patients régulièrement suivis par leur médecin traitant [29]. Pour y parvenir, il est primordial de promouvoir la formation médicale continue des médecins de première ligne. La sensibilisation des patients à une meilleure compréhension de leur maladie et à l'urgence (par exemple, définir les critères d'exacerbation d'une pathologie chronique motivant une consultation urgente, développer la culture médicale du patient en abordant des thèmes d'urgence), ainsi qu'à une observance thérapeutique et aux bénéfices d'un suivi médical régulier pourraient contribuer à réduire le recours aux urgences pour des motifs non urgents. En outre, la délégation de certaines tâches (éducation, prévention, conseils, certaines prescriptions) au personnel paramédical pourrait avoir un impact sur la réduction de l'engorgement des SU [26].

Le tri aux urgences constitue aussi une des solutions intéressantes mises en place dans plusieurs pays développés [29], mais il fait encore défaut dans plusieurs pays de la Région de la
Méditerranée orientale [31]. Une étude réalisée à Bahreïn a mis en évidence que le tri effectué par des médecins a permis d'orienter plus de la moitié des patients $(54,4 \%)$ qui consultaient les SU vers une consultation externe ou leur domicile [28]. La plupart des études s'accordent pour affirmer que si le tri est effectué par un médecin, une réduction à la fois du délai d'attente et du nombre de patients partis sans être vus peut être obtenue [31]. D'autres recommandent la « seniorisation »des urgences qui aurait un impact positif sur la diminution des prescriptions d'examens complémentaires et permettrait une meilleure orientation du patient [32]. Dans cette optique, certains pays tels que la Tunisie et le Sultanat d'Oman ont fait le choix depuis la dernière décennie de faire de la médecine d'urgence une spécialité médicale, ce qui permettrait une amélioration de la qualité des soins dans les SU $[31,33]$. Le renforcement des effectifs médicaux et paramédicaux s'impose durant les plages horaires où l'afflux aux urgences est le plus élevé, en l'occurrence le soir [34].

La création de la voie express ou fast track qui permet d'examiner les patients les moins graves en 30 minutes, leur permettant ainsi de regagner rapidement leur domicile, a un double avantage : le désencombrement rapide de la salle d'attente et un gain de temps pour les patients les plus gravement atteints [35]. Cependant, selon Nash K., cette solution risquerait d'augmenter la fréquentation des urgences [36].

Les études menées actuellement sur l'encombrement des urgences suggèrent de façon claire que le détournement du flux d'entrée pour les patients non urgents ne résout que partiellement le problème. En fait, le problème réside plutôt dans le débit de sortie, notamment dans l'incapacité du système hospitalier à transférer les patients des urgences vers les services d'hospitalisation [20].

Par ailleurs, la mise en place d'un dossier médical informatique unique pour chaque patient, accessible dans tous les services où passera le malade, a fait la preuve d'une certaine efficacité dans la réduction de l'engorgement des urgences [37].

D'autres solutions ont été testées, telles que les maisons médicales de garde ou les walk-in centers. L'efficacité de ces centres de conseil rapide et de traitement, ouverts $24 \mathrm{~h} / 24$ et qui regroupent infirmières et/ou médecins ne fait pas l'unanimité [38]. En France, l'impact des maisons médicales de garde est difficile à évaluer. Le temps d'attente aux urgences diminue pour les patients qui présentent des pathologies peu graves (CCMU 1 et 2), mais seulement lorsqu'une organisation précise et intégrée dans le fonctionnement des urgences a été mise au point [39].

\section{Conclusion}

Si certaines pratiques semblent désengorger les services d'urgence, les solutions consisteraient plutôt à mettre en place simultanément plusieurs bonnes pratiques dans le cadre d'une démarche qualité, et à créer une meilleure complémentarité entre les services d'urgence et les structures de première ligne publiques et privées.

Une réorganisation doit être discutée avec les divers partenaires du système de santé en tenant compte de la demande et des besoins de la population, mais également en intégrant la rationalisation du fonctionnement et la maitrise des coûts, et en valorisant la qualité de la formation des futurs praticiens ambulatoires. 


\section{Références}

1. Rehmani R, Norain A. Trends in emergency department utilization in a hospital in the Eastern region of Saudi Arabia. Saudi Medical Journal, 2007, 28:236-240.

2. Gentile $\mathrm{S}$ et al. Nonurgent patients in the emergency department? A French formula to prevent misuse. BMC Health Services Research, 2010, 10:66.

3. Chahed MK, Somrani N, Achour H. L'offre et I'utilisation des services d'urgence en Tunisie : principaux résultats d'une enquête nationale exhaustive [Availability and use of emergency services in Tunisia: main results of a comprehensive national survey]. La Revue de Santé de la Méditerranée orientale, 2001, 7(4/5):805-811.

4. Scheefer S, Mancuso G, Stein-Mergen M. Augmentation de I'affluence des patients aux urgences du Centre Hospitalier de Luxembourg. Qui sont-ils? Pourquoi viennent-ils? [Increase in the inflow of emergency patients at the Central Hospital of Luxembourg. Who are they? Why do they come?]. Bulletin de la Société des Sciences Médicales du Grand-Duché de Luxembourg, 2005, 1:91-106.

5. Comprendre les temps d'attente dans les services d'urgence: Qui utilise les services d'urgence et quels sont les temps d'attente? [Understanding Emergency Department Wait Times: Who is Using Emergency Departments and How Long Are They Waiting?]. Ottawa, Institut canadien d'information sur la santé (ICIS), 2005 (http://secure.cihi.ca/cihiweb/products/ Wait_times_f.pdf, consulté le 25 octobre 2011).

6. Capewell S. The continuing rise in emergency admissions. British Medical Journal, 1996, 312(7037):991-992.

7. Burt CW, McCaig LF. Trends in hospital emergency department utilization. United States, 1992-99. Vital Health Statistics13, 2001, 150:1-34</unknown>

8. Elkum $\mathrm{N}$ et al. Which patients wait longer to be seen and when? A waiting time study in the emergency department. Eastern Mediterranean Health Journal, 2009, 15:416-424.

9. Al-Shaqsi SZ. EMS in the Sultanate of Oman. Resuscitation, 2009, 80:740-742.

10. Cohen AD et al. Vardy Utilization of emergency department services by the Bedouin population in southern Israel DA. The Scientific World Journal, 2007, 7:330-335.

11. Baubeau D, Carrasco V. Motifs et trajectoires de recours aux urgences hospitalières [Reasons for using hospital emergency departments and referral pathways]. Direction de la recherche, desétudes, del'évaluation et des statistiques(DREES), Ministère des Affaires sociales, du travail et de la solidarité/Ministère de la Santé, de la famille et des personnes handicapées, 2003 (Études et Résultats n ${ }^{\circ}$ 215) (http://www.sante.gouv.fr/IMG/ pdf/er215.pdf, consulté le 25 octobre 2011).

12. Herzlich C. Types de clientèle et fonctionnement de I'institution hospitalière [Types of Patients and Hospital Procedures]. Revue française de sociologie, 1973, XIV (Numéro spécial « Sociologie de la médecine »):41-59.

13. Shah NM, Shah MA, Behbehani J. Predictors of non-urgent utilization of hospital emergency services in Kuwait. Social Science \& Medicine, 1996, 42:1313-1323.

14. Al-Shammari SA. Use and abuse of emergency services in Riyadh health centres, Saudi Arabia. Saudi Medical Journal, 1991, 12:489-493.

15. Baubeau D, Carrasco V. Les usagers des urgences: premiers résultats d'une enquête nationale [Emergency department users : preliminary findings of a national survey]. Direction de la recherche, des études, de l'évaluation et des statistiques (DREES), Ministère des Affaires sociales, du travail et de la solidarité/Ministère de la Santé, de la famille et des personnes handicapées, 2003 (Études et Résultats $\mathrm{n}^{\circ}$ 212) (http://www. sante.gouv.fr/IMG/pdf/er212.pdf, consulté le 25 octobre 2011).

16. Gentile $\mathrm{S}$ et al. Attitudes et comportement des usagers face à une urgence réelle ou ressentie [Attitude and behaviour of users when faced with a medical emergency, either real or perceived as urgent]. Santé publique, 2004, 16(1):63-74.

17. Gentile $S$ et al. Les consultants des services d'urgence relevant de la médecine générale : analyse de nouveaux comportements de santé [The consultants of emergency services within general practice: analysis of new health behaviors]. Journal européen des urgences, 2007, 20(1S):138.

18. Recours urgents ou non programmés en médecine générale dans les Pays de la Loire. (Urgent or unplanned referrals in general practice in the Loire region]. Nantes, Observatoire régional de la santé des Pays de la Loire (ORSPL), 2007.

19. Abdallat AM, al-Smadi I, Abbadi MD. Who uses the emergency room services? Eastern Mediterranean Health Journal, 2000, 6:1126-1129.

20. Kierzek G, Pourriat JL. De nouvelles missions pour les services d'urgences? [New missions for emergency department]. La Revue du Praticien, 2009, 59:1265-1277.

21. Sarver JH, Cydulka RK, Baker DW. Usual source of care and nonurgent emergency department use. Academic Emergency Medicine, 2002, 9:916-923.

22. Ladner $\mathrm{J}$ et al. Les patients auto-référés dans les services hospitaliers d'urgences : motifs de recours et comportements de consommation de soins [Patients who refer themselves to hospital accident and emergency departments: reasons for consulting and how they follow their treatment]. Pratiques et Organisation des Soins, 2008, 39(1):33-42.

23. Cohen J. Accident and emergency services and general practice-conflict or cooperation? Family Practice, 1987, 4:8183.

24. Mahfouz AA et al. Primary health care emergency services in Abha district of southwestern Saudi Arabia. Eastern Mediterranean Health Journal, 2007, 13:103-112.

25. Unsal A et al. Use of emergency departments by elderly patients in a city of Western Turkey. European Journal of Emergency Medicine, 2007, 14:125-129.

26. Cour des Comptes. Les urgences médicales: constats et évolution récente [Medical emergencies: findings and recent developments] In Le Rapport public annuel. Première partie - Les observations des juridictions financières. [Annual Public Report. Part I - Observations from the financial juridictions]. Paris, La documentation Française, 2007, 313-347.

27. Dale J et al. Primary care in the accident and emergency department: I. Prospective identification of patients. British Medical Journal, 1995, 311:423-426.

28. Fateha BEA, Hamza AY. Impact of triage in accident and emergency departments in Bahrain. Eastern Mediterranean Health Journal, 2001, 7:790-798.

29. Holroyd BR et al. Impact of a triage liaison physician on emergency department overcrowding and throughput: a randomized controlled trial. Academic Emergency Medicine, 2007, 14:702-708.

30. Jerius $\mathrm{M}$ et al. Inappropriate utilization of emergency medical services at Prince Ali Military Hospital. Journal of the Royal Medical Services, 2010, 17:32-35.

31. Qureshi NA. Triage systems: a review of the literature with reference to Saudi Arabia. Eastern Mediterranean Health Journal, 2010, 16:690-698. 
32. Gerbeaux $\mathrm{P}$ et al. Impact de la «seniorisation » sur les prescriptions d'examens de biologie dans un service d'accueil et des urgences [Effect of the "seniorization" of the medical staff of an Emergency medical service on the prescriptions of biological tests]. Annales Francaises d'Anesthésie et de Réanimation, 2000, 19(1):62-66.

33. Al-Azri NM. Emergency medicine in Oman: current status and future challenges. International Journal of Emergency Medicine, 2009, 2:199-203.

34. Lovis A et al. Quelle organisation pour les services d'urgence des hôpitaux périphériques de demain ?L'exemple del'hôpital de Nyon [How to organize the emergency department of primary care hospitals in the future? Nyon's regional hospital experience]. Revue médicale Suisse, 2007, 3(133): 2584-2586.

35. Péricaud P. Évaluation des solutions à l'engorgement des urgences en France et à l'étranger (Royaume-Uni, Canada, ÉtatsUnis). Revue internationale de la littérature [thèse] [Evaluation of solutions to emergency department overcrowding in France and abroad (United Kingdom, Canada and United States).
A review of international literature ]. Paris, Université Paris Descartes, 2009.

36. Nash $\mathrm{K}$ et al. Evaluation of the fast track unit of a university emergency department. Journal of Emergency Nursing: JEN, 2007, 33:14-20.

37. Debaud S. Informatisation del'accueil des urgences: une première démarche vers l'assurance qualité [thèse] [Computerization of the emergency department: a first step towars quality assurance]. Paris, Université Paris Descartes, 1997.

38. Salisbury $\mathrm{C}$ et al. The impact of co-located NHS walk-in centres on emergency departments. Emergency Medicine Journal, 2007, 24:265-269.

39. Lemasson H, Jasso-Mosqueda G, Chicoye A. Évaluation des 5 maisons médicales de garde financées par Le FAQSV Ile de France. Rapport final-synthèse [Evaluation of 5 "maisons médicales de garde" (out-of-hours general practice walk-in centres) funded by the FAQSV Ile de France. Final Report - Synthesis]. AREMIS Consultants, 2005, 15 p. (http://pds78.org/Pdf/synthese_ volet2.pdf, consulté le 26 octobre 2011).

\section{Replacement of mercury thermometers and sphygmomanometers in health care}

Replacement of mercury thermometers and sphygmomanometers in health care is a short guide designed to provide stepby-step instructions for the safe substitution of non-mercury thermometers and sphygmomanometers in health-care settings. It identifies the resources available to provide confidence that the substituted products will provide equivalent accuracy and comparable clinical utility, while protecting health-care workers and the environment. It is designed for professionals responsible for institutions or ministries desiring to switch to safer non-polluting technologies in health care.

This guide is also an output of a global mercury-free health-care initiative in which WHO is engaged. This global initiative aims to promote the substitution of mercury-based medical devices with safe, affordable, accurate alternatives around the world. The global mercury-free health-care initiative has documented mercury substitution in dozens of countries. It has also produced a series of additional resources for health professionals, health-system managers and government officials that can be useful in developing and implementing policy and strategies for mercury substitution in the health sector

Further information about this and other WHO publications is available at: http://www.who.int/publications/en/ 